\title{
Carnets
}

Revue électronique d'études françaises de l'APEF

Deuxième série - 10 | 2017

Pratiques de l'errance, vécus de la mémoire

\section{Anachronies, oubli et délire de théatralisation}

Le Sankofa et la mémoire empêchée chez Léonora Miano

\section{Ferdulis Zita Odome Angone}

\section{(2) OpenEdition}

Journals

Édition électronique

URL : http://journals.openedition.org/carnets/2200

DOI : $10.4000 /$ carnets.2200

ISSN : 1646-7698

Éditeur

APEF

Référence électronique

Ferdulis Zita Odome Angone, "Anachronies, oubli et délire de théatralisation », Carnets [En ligne], Deuxième série - 10 | 2017, mis en ligne le 30 avril 2017, consulté le 02 mai 2019. URL : http:// journals.openedition.org/carnets/2200; DOI : 10.4000/carnets.2200

Ce document a été généré automatiquement le 2 mai 2019.

\section{(c) (i) \&}

Carnets est mis à disposition selon les termes de la licence Creative Commons - Atribution - Pas d'utilisation commerciale 4.0 International. 


\title{
Anachronies, oubli et délire de théatralisation
}

\author{
Le Sankofa et la mémoire empêchée chez Léonora Miano
}

Ferdulis Zita Odome Angone

$1 \mathrm{Au}$ sein des littératures hybrides, l'effet d'anachronie rend compte d'une identité disloquée. Tout part d'une histoire fragmentée, discontinue et composite où l'ombre d'un passé refoulé influe sur le présent et semble vouloir régenter le futur du narrateur par la multiplication des séquences mémorielles généralement analeptiques. Cela peut se traduire dans la fiction par diverses notions de rétrospective telles que les souvenirs, la répétition, la redondance, l'allusion à des évènements ou des faits énoncés avec antériorité et toutes les références extratextuelles historiques introduites dans la trame. A cet effet, Sartre exposait déjà que «la temporalité est évidemment une structure organisée et ces trois prétendus "éléments" du temps : passé, présent, avenir, ne doivent pas être envisagés comme une collection de « data » (...) mais comme des structures d'une synthèse originelle » (Sartre, 1943/2011: 142).

2 Ainsi, l'effet d'anachronie permettrait de comprendre l'impression de décalage, de déjàvu, d'inquiétante étrangeté que suscitent certaines séquences (évènements, lapsus, actes manqués, flashs back, etc.) projetées dans un temps à rebours échappant à toute contemporanéité. Une anachronie peut être notamment, «un mot, un évènement, une séquence signifiante sortis de "leur" temps, doués du même coup de la capacité de définir des aiguillages temporels inédits, d'assurer le saut ou la connexion d'une ligne de temporalité à une autre $»^{1}$. Nous retenons dès lors que les analepses ${ }^{2}$ réitératives ne sont pas toujours des digressions anodines ni de simples allusions narratives, car leur fréquence et leur pertinence s'apprécie en fonction de leur aspect singulatif, anaphorique, répétitif ou itératif ${ }^{3}$. Notre propos est d'analyser le concept du Sankofa illustré par Epupa, le personnage identifié comme fou dans les premiers romans de Léonora Miano, selon la perspective d'Edouard Glissant (2002). D'après l'essayiste antillais, la folie ou le personnage souffrant de cette pathologie met en évidence «la virtualité non réalisable $»^{4}$ tributaire du 《délire de théâtralisation ${ }^{5}$. Le délire de 
théâtralisation exhumerait en réalité la mémoire empêchée ${ }^{6}$ et serait ainsi l'émanation symptomatique d'une amnésie collective productrice de sens.

Le trinôme : Sankofa, délire de théâtralisation et Epupa

3 Le SANFOKA est un concept du peuple Akan, un peuple géographiquement situé en Afrique de l'Ouest. Le mot "sankofa" est une conjonction de morphèmes des verbes : - « san » (reviens); - « ko » (va); - "fa » (regarde, cherche et prend). Comme un exercice d'autocritique, le concept enseigne sur la nécessité de puiser au-dedans de soi pour avancer vers la bonne direction. Cela implique, de ce fait, un retour vers les racines, les origines, pour ressortir le meilleur de ce que le passé enseigne afin d'explorer tout le potentiel pour aller de l'avant. Sur le plan symbolique, le SANKOFA représente un oiseau mythique en position horizontale, de façon hiératique, la tête "paradoxalement" tournée vers l'arrière avec en son bec un œuf (symbole de futur). Miano illustre le concept akan ainsi qu'il suit :

Sanfoka est le nom d'un oiseau mythique. Il vole vers l'avant, le regard tourné en arrière, un œuf coincé dans son bec. L'œuf symbolise la postérité. Le fait que l'oiseau avant en regardant derrière lui signifie que les ressorts de l'avenir sont dans le passé. Il ne s'agit pas de séjourner dans l'ancien temps, mais d'en retirer des enseignements. (Miano, 2009 : 225).

4 Voyons à présent le rapport entre le Sankofa et le personnage Epupa, parallélisme qui apparaît déjà dès le premier roman de Miano (2005), bien que son héroïcité culmine dans son troisième ouvrage (2009). Figure paradigmatique de la «névrose collective» en région subsaharienne, Epupa représente les tumultes d'une société convulsive qui sombre dans l'amnésie. Epupa est perçue comme folle aussi bien par ses voisins, par la collectivité -peut-être moins par le lecteur averti- que par les institutions qui la marginalisent :

(...) c'est une folle (...) Elle s'appelle Epupa (...)- déclare Epésè. (Miano, $2005:$ :211)

Sa folie s'identifie par l'étrangeté d'un discours délirant en opposition à une norme sociale et idéologique aliénante standardisée (mue par des modèles erronés et intériorisés). Epupa serait le symbole d'un pathos collectif et le spectre malveillant de l'angoisse général qui gangrènent les peuples subsahariens endormis dans une dynamique de l'inertie. Notre personnage serait donc la manifestation virale d'une catharsis commune en délire. Ici, nous observons une relation dialogique imprimée par un sentiment de décalage, d'inquiétante étrangeté entre la société et Epupa. La pathologie apparente de cette dernière serait le reflet de cette société-là dans un processus d'amnésie extrême qu'elle n'arrive plus à voir en Epupa le reflet même de sa propre démence $^{7}$. La folle symboliserait ainsi le symptôme d'une angoisse commune, refoulée, qui remonte. Ce familier angoissant qui revient, dans le cas d'Epupa, expulserait probablement les éléments traumatiques évanouis dans l'inconscient collectif du passé irrésolu.

6 Face à un déficit d'histoire ou à cause d'une version historique officielle incongrue, Epupa expulse le double malveillant de la société qui la perçoit comme agent délirant. De sorte que par voie de parallélisme, la société postcoloniale traumatisée qui, d'une certaine façon se reconnaît en Epupa, préfère évincer le camouflet de l'oubli en contournant provisoirement cet angoissant qui remonte. Cette réaction se présente comme le Moi désemparé du collectif dont l'autodéfense préfère éluder ainsi sa propre aliénation en identifiant Epupa comme folle au lieu d'exhumer le malaise ambiant. En réalité, la lecture des textes va démontrer que, loin de délirer, notre personnage retourne à l'expérience princeps pour rouvrir les blessures d'un passé dissimulé, afin de « tisser une histoire » et 
«ressusciter une époque dont le souvenir n'existe plus» (Miano, 2013), l'apparente absence de la matière n'étant pas vacuité mais constance, consistance, persistance et conscience du passé oublié ou évanoui.

Ayané vit arriver Epupa, qu'elle n'avait pas revue depuis des mois. (...) Elle était pieds nus (...) elle parla, presque pour elle-même. - Depuis l'abysse, ils nous regardent. Ils parlent mais nous n'écoutons pas. Pourtant, ils ne furent jamais des ossements desséchés, sans conscience... (...) - Ceux qui sont morts ne sont jamais partis! $!^{8}$ Ce n'est pas d'aujourd'hui que le sang des nôtres a rougi l'aurore, et nous sommes captifs de leur tourment... Je dis Sankofa! Pour qu'ils habitent notre mémoire. Sankofa! Pour que le passé nous enseigne qui nous sommes à présent. Sankofa! Pour résider en nous-mêmes, mais aussi hors de nous, réconciliés avec nos peines. Sankofa! Pour nous délivrer de toute haine (Miano, 2009: 160-162).

En effet, Epupa «la folle la plus célèbre de Sombé », [mis à part ses signes évidents de trouble mental - elle étrangle son fils, un nourrisson en plein jour (Miano, $2006: 22)]$, elle était étudiante :

La vox populi disait qu'Epupa « était devenue folle à force d'étudier l'Histoire », une précision révélatrice car l'origine de sa démence peut trouver là une explication liée à l'Histoire.

\section{"L'histoire, la mémoire et l'oubli » à la croisée du « nouvel ordre narratif »}

8 L'oubli par évanouissement des empreintes corticales n'épuise pas le problème de la mémoire empêchée puisque divers oublis sont dus à l'impossibilité d'accéder aux éléments enfouis dans la mémoire. Selon les psychanalystes, l'identification d'une image du passé peut contribuer à favoriser le retour à l'expérience princeps pour ainsi désamorcer la bombe latente. C'est pourquoi, face à l'impuissance d'extirper le trauma, l'organisme cherche - sous diverses formes malignes - d'autres issues de secours pour substituer ses troubles réactionnels (les lapsus, les souvenirs-écrans et autres actes manqués). De ces troubles, Fanon dira qu'ils persistent [longtemps], attaquant massivement le moi, et laissant presque toujours comme séquelle une fragilité pratiquement discernable à vue d'œil. Ce qui de toute évidence hypothèque l'avenir $\mathrm{d}[\mathrm{u}]$ malade (Fanon, 1961, 1968/2002 : 242). Ainsi, face à l'aberration d'une société décadente qui culmine dans l'oubli, Epupa est devenue folle. Face à une Histoire sectionnée, segmentée, fragmentée, fractionnée, fracturée que lui enseignent les canons officiels et dont les épisodes déterminants sont soient censurés, soient tus, Epupa a opté pour la folie comme le meilleur des langages. Face à une mémoire tronquée par un discours dominant qui masque (ou qui refuse de regarder) la réalité, Epupa souffre de ce que Glissant appelle le « tourment d'histoire » exprimé par « la virtualité non-réalisable » :

Le mal continental était spirituel. (...) Le péché continental résidait dans l'oubli. Ceux des déportés du trafic triangulaire qui n'avaient pas peuplé, fécondé les Amériques, avaient péri dans les flots. (...) Ils réclamaient la mémoire. (...) Leur peine s'infiltrerait dans l'existence des peuples. Dans l'ensevelissement, les différentes populations déportées lors du Passage du milieu s'étaient mêlées. Elles ne connaissaient plus qu'une seule langue, charriant l'écho de toutes celles qu'elles avaient parlées. (...) Chaque fois que les liens familiaux se défaisaient, chaque fois qu'on se dressait contre son semblable, l'Histoire se répétait. Douloureusement, impitoyablement, dans un entre-soi subsaharien. La traite négrière était à inscrire au patrimoine du genre humain (Miano, 2009: 198 et 208). 
9 Epupa vit l'histoire comme un/e tourment/e et comme résidu symptomatique d'un passé refoulé. La virtualité ne se retrouve pas réalisée en elle par la rupture de la dépendance totale du circuit de production aliénante qui s'illustre par son "délire ». C'est à ce titre que tout comme Epupa, tous ceux qui renonceront au diktat du discours dominant seront perçus comme déviants, délirants, fous. Nous pouvons comparer le délire de théâtralisation d'Epupa avec le délire de représentations des institutions publiques et de la société en général. Dans un premier temps, ils reconnaissent le délire d'Epupa comme souffrance, avant de la repousser lorsqu'elle met à nu l'histoire que le collectif ne veut/ peut pas assumer. L'accusation que lance Epupa concernant les "sans sépulture » péris lors du Passage du Milieu $u^{9}$ est ressentie comme une injure insupportable par la communauté qui préfère la repousser pour « pur délire » :

Sa voix se faisant plus puissante à mesure qu'elle parlait, comme une tornade qui fondait sur vous avant que l'idée de fuir ne soit clairement formée dans votre esprit. Les gens du quartier la connaissaient bien. Toute la ville la connaissait (...) (Miano, 2009 : 162).

10 Des flashs surgissent, quelques personnages perçoivent un message, un langage disséminé qui ne leur apparaît pas encore avec lucidité. Serait-ce des actes manqués qui leur échappent? Des lapsus? Ce sont des syllabes éparses d'un nom qui commence dès le premier roman (2005) et se dénoue dans le troisième roman de Miano (2009). Un nom symbolique, SANKOFA, vient de façon sibylline, par morphèmes sans que les intéressés ne sachent bien à quoi il se réfère. Un nom parfois déformé, toujours énigmatique dont on n'arrive pas encore à percer les mystères, ni à détecter le lien et les connexions immédiates. Un nom à la fois symptôme et antidote :

La femme [Ayané] ouvrit les yeux. Le sommeil n'avait duré que quelques minutes imprimant en elle un malaise. Il lui avait semblé entendre des voix, rauques d'avoir trop gémi. Pourtant, il lui était impossible de se rappeler ce qu'elles avaient dit. Il ne lui restait en mémoire que des plaintes étouffées, un bruit de chaînes traînées sur un sol en bois. Des sons hachés qu'elle ne parvenait pas à assembler pour en tirer un sens: San... San Ko... Ko... Elle ne s'expliquait ni cela, ni cette odeur marine qui flottait dans l'air, comme si toute la vase de la Tubé avait été remuée, transportée dans la pièce (Miano, $2009: 17-18)$.

$11 \mathrm{Au}$ lieu d'entendre, "San-Ko-Fa" avec discernement, les personnages «délirants » perçoivent ses syllabes segmentées sans pouvoir l'associer au nom adéquat, signe que l'anciennement familier est en train de remonter. Hormis chez Ayané, cela apparait aussi chez Epa, un autre sujet « délirant » :

Dans l'état d'épuisement où j'étais, j'aurais dû dormir d'un sommeil sans rêves. Ce ne fut pas le cas. (...) Sans qu'aucun indice précis me le confirme, j'ai eu le sentiment d'avoir été projeté dans un autre espace temporel. (...) Eyia se trouvait là, assis au milieu d'un groupe de personnes sans visage. (...) Comme je me jetais à ses pieds pour tenter de lui ôter sa chaîne, il s'est mis à secouer la tête, avant d'ajouter : - Frère, je reconnais bien ta fougue et ton entêtement, mais tu perds ton temps. Fais ce que je te dis. Parmi ceux qui m'entourent, certains sont des Ekus, razziés sur nos terres il y a bien longtemps, et dont l'histoire est tue. Ils se joignent à moi pour te demander de leur restituer leur place au sein de la communauté. Tu ne pourras nous rétablir dans la chair, puisque nous ne sommes plus, physiquement. Cependant, en refusant que les vivants d'aujourd'hui soient, eux aussi, arrachés à leur matrice et enfermés dans l'absurde, tu détruiras nos chaînes.

Je n'ai pas eu le temps de dire à Eyia que je ne comprenais pas un traître mot à ce qu'il racontait. Une femme sans visage a bondi vers moi, faisant claquer sa chaîne, entraînant avec elle quelques-uns de ses compagnons. Elle a posé une main froide sur mes lèvres, tandis que ceux qui l'entouraient se mettaient à hurler : Sankofa! 
Sankofa! (...) Qui étaient ces générations d'Ekus? De quand datait leur disparition? ( ibid. : $67-69) .^{10}$

12 Aussi tout délire doit-il être interprété comme révélation d'une empreinte, identification d'un indice, manifestation d'un nom, émission d'un son presque oublié et dont le sens demeure inaccessible, mais qui cependant connectera les concernés à ce passé camouflé. Epupa a localisé la trace de cet épisode demeuré en suspens, mais elle ignore ce savoir qui l'habite et c'est cette ignorance qui la rend folle. Il s'agit là d'une mission prophétique qui s'exprime par sa transe :

Epupa s'était éclipsée sans se faire remarquer. Son destin l'attendait. (...) Elle ouvrait sur le monde des yeux harcelés d'images illisibles, qu'elle ait cru les voir dans l'eau ou qu'elle les ait rêvées. Au début, tout s'embrouillait. La jeune femme se surprenait, sans l'avoir prémédité, à haranguer les foules, à prêcher, à mettre en garde. Se trouvant quelque part, elle voyait distinctement que des évènements se produisaient ailleurs, en ces temps comme en d'autres. Un tumulte s'emparait d'elle, la poussant à invectiver de pauvres gens qui ne comprenaient rien à ce qu'elle disait (...) C'était alors qu'on l'avait qualifiée de folle, qu'on s'était mis à rire d'elle, à l'injurier. (...) Non seulement elle voyait des choses qu'elle n'avait aucune envie de contempler, prononçant des mots qu'une part d'elle-même, dorénavant muette, savait n'avoir jamais conçus, mais elle était atrocement seule. Une paria. Quelqu'un qu'on ne mettait pas à mort uniquement parce qu'on pensait que les communautés engendraient naturellement des êtres troubles, devant incarner leur face sombre (ibid. : 256-258). (...) L'ayant instruite de choses essentielles, elles [les femmes sans visage et sans nom] lui avaient demandé de les incarner à la surface.

- Là-haut, avaient-elles dit, notre nom s'est perdu. Laisse-nous habiter ta chair pour nous faire entendre. Ensuite, tu seras libre. Prends ceci. Nous avons tissé cette étoffe de cruor au fil des siècles, espérant le passage d'une âme ouverte... (ibid. : 260).

13 Dorénavant, il est connu que les gestes ainsi que les actes manqués de la communauté crient le déficit d'histoire, une histoire hystérique camouflée par la synchronie d'une amnésie collective. Il est question ici précisément d'hommage méritoire jamais rendu à ceux qui périrent dans le plus grand anonymat et dans l'indifférence la plus abjecte durant le Passage du Milieu. Ces «sans sépulture » déterminés à faire entendre leur « voix », exigent un hommage " proportionnellement égal » à la cruauté avec laquelle leurs corps périrent dans le cynisme des océans anthropophages du cénotaphe bleu durant la Traite Négrière. Des morts et disparus dont on parle très peu -ou presque jamais- aussi bien dans les manuels scolaires que dans les commémorations et divers hommages - encore embryonnaires sur le Continent- alors que, paradoxe, l'Afrique subsaharienne dont ils sont issus, accorde pourtant une place prépondérante au culte cosmogonique des Morts qui, jamais, ne le sont :

- Maintenant, il suffit! Je le dis, et le redis. Suffit de faire semblant, de toujours accuser les autres. Ils ont leurs torts, et leurs mains sont souillées du sang dont ils se sont abreuvés. Mais quoi qu'ils aient pu faire, et quelles qu'aient pu être les manipulations ourdies par eux contre nous, ils ne peuvent porter les crimes qui sont les nôtres.

Pouvons-nous continuer à prétendre que des millions de nos fils nous furent arrachés, sans la moindre complicité sur place ${ }^{11}$ Confessons la faute! Prenons-en notre part. (...) Il est temps de reconnaître que nous avons participé à notre propre saignée... Si nous n'admettons pas les noirceurs du passé, saurons-nous nous défaire de celles qui nous étreignent encore si vigoureusement? Voici ces jours nos fils en route pour la guerre. ... Tandis que nous quémandons la culpabilité de l'Occident, à qui nos enfants demanderont-ils réparation? - poursuit Epupa (Miano, 2009: 212-213). 
On peut observer ici un certain tragique de la vie et une déformation grotesque des sociétés subsahariennes où l'absurde s'observe dans les figures incongrues et spectrales de la foule. Dans la prose de Miano, il se dégage ici les promesses d'une esthétique du délire où la folie apparente débusque la folie vraie devenue le moule de prescriptions communes à une société amnésique. En parcourant Les aubes écarlates, en effet, il nous surgit un doute sur les morts. Sont-ils vraiment morts? On se demande aussi si les vivants le sont-ils vraiment car l'ombre des défunts continue de hanter ces derniers, une conséquence probable des hantises du passé irrésolu (Rousso: 1998). La difficulté de séparer les morts des vivants, le passé du présent met en relief l'absence de deuil. La carence du travail de mémoire rappelle bien la difficulté de repos des morts qui ne purent jamais reposer en paix, c'est pourquoi ils sont décidés à perturber l'apparente quiétude des sur/vivants :

Nous sommes la faille, le gouffre. Notre absence est le cœur de ce continent. Nous sommes la mémoire proscrite, la honte muette. Ceux qui nous donnent le dos depuis des générations ignorent que nous sommes l'air qu'ils respirent. Nous sommes la suffocation, l'étouffement. Nous sommes l'atmosphère brûlant mais sans chaleur. Nous ne caressons, ni n'apaisons. Nous sommes le démembrement, l'écartèlement, le grand égarement. Notre âme s'est faite rancunière au fil des âges $(2009: 38) .^{12}$

Les morts sont zombifiés par leur malemort (Glissant, 1975/1997). Cela rend impossible leur rupture d'avec les vivants. Nous savons désormais que l'acmé du récit se présente comme une spirale qui explore ce maelström au sein duquel un discours délirant s'est converti en dominant. Le langage mystérieux, « déviant », parfois incohérent d'un tel ou d'une telle autre, n'était que la rémanence d'un symbole ancien, demeuré en suspens, tant que les morts étaient enveloppés dans le tourbillon de l'oubli. Au niveau esthétique, Miano entrecoupe le texte d'interludes dénommés «Exhalaisons» (2009: 11-14, 38-40, 122-123 et 189-191). Elle souligne ainsi le parallélisme d'un pamphlet «caché » où les morts marquent/ponctuent de façon intermittente le souffle des vivants et «s'exhument » pour réclamer rétrospectivement leurs « droits » :

Peut-être nous entendras-tu, toi dont la conscience ne cesse de remuer l'intangible. Tu pressens, plus que tu ne saurais l'expliquer, que le sens des choses est également au-delà du visible. Alors, peut-être entends-tu. (...) Comme le vent réside entre ciel et terre, nous sommes. Suspendus sans être accrochés. Seulement en suspens. Notre route fut tranchée. Non pas notre existence, puisque la vie ne s'interrompt pas. Toi dont la conscience cherche à sonder l'intangible, tiens la réponse à tes questions : nous sommes l'absurde quotidien. Nous sommes la haine du frère, la haine de soi. Nous sommes l'impossibilité, l'entrave au jour qui vient. Nous en avons emprisonné les contours au creux de notre main immatérielle (...) (ibid.: 11-12). ${ }^{13}$

Nous pouvons remarquer que les anaphores et les allitérations sont les figures stylistiques de plus grande usance dans ces fragments. La répétition du même mot, de la même phrase et du même syntagme sémantique et/ou phonique rythment l'obsession et provoque un effet accumulatif qui souligne la synergie autour de l'impulsion et de la convulsion du discours. Le "nous", avec lequel s'identifient les "sans sépulture ", acquiert une forme impérative et emphatique pour renforcer la densité agglutinante d'un collectif compact et innombrable presque vertigineux, déterminant et déterminé. Leur discours culmine ainsi dans le dernier interlude :

Comprends ce que nous disons: le linceul qui ne fut pas tissé voile la face du Continent. Son ombre est sur les jours de cette terre. Nous sommes las de sévir. 
Épuisés de châtier. Qu'on nous donne la route. Qu'est-ce qu'une stèle, sur ce sol qui regorge de richesses ? Qu'est-ce qu'une journée du souvenir, pour ceux dont la généalogie se confond avec le premier matin du monde ? Qu'on nous donne la route. Nous ne sommes plus la joie. (...) Nous voudrions la paix enfin. Accéder, nous aussi, à cet autre monde où les trépassés deviennent des figures tutélaires. Que notre arrachement n'ait pas été vain. Que nos déchirures soient lues par le monde, pour être, dans la conscience du monde, au-delà de l'effroi, l'expérience de chacun. Qu'on nous donne la route. (...) Si même nous voulions caresser les vivants, notre main ne pourrait que flétrir leur existence. Si même nous voulions apaiser les souffrances, notre main ne ferait que calciner leur chair. Qu'on nous donne la route. Sache : il ne nous agrée pas de devenir un capital victimaire. Il ne nous agrée pas qu'on s'abaisse à demander réparations sonnantes et trébuchantes (...). Il ne nous agrée pas qu'on montre du doigt les fautifs, quand on est soi-même l'auteur d'un crime moral. Nous voyons une injure (...) Nous voyons du mépris (...) Nous étions les vaillants et nous étions les braves. Nous étions la beauté, et la vitalité. Nous étions la promesse, et la fertilité. Nous étions le courage, nous étions la cadence. Nous étions l'endurance et l'imagination. (...) Tandis que nos corps s'affaissent sous les vagues, nous devînmes la longue expiration qui te parvient ce jour, à toi qui sondes l'intangible, en quête de sens. Si le rapport au monde jaillit de l'appartenance à un espace donné, s'il ne peut se concevoir qu'à partir de soi, rien n'est possible pour ceux qui s'amputent d'un morceau d'eux-mêmes. Il n'est nulle part de puissant qui foule aux pieds la mémoire de ses défunts... (ibid.: 189-191). ${ }^{14}$

17 Les interludes constituent aussi des espaces propices pour une praxis lyrique. En abandonnant un type de narration descriptif ou un discours qui se développerait à partir de plusieurs perspectives distribuées chez divers personnages selon le jeu des points de vue, ici nous avons une pluralité de voix qui offre une sonorité univoque comme pour mieux essaimer la lamentation et souligner la vibration active qui détermine le destin des morts d'avec celui des vivants. Car, comme on peut le noter, les écueils des vivants ont un effet contrapuntique d'avec le tumulte des "sans sépulture ». L'irruption d'un langage poétique offre un dialogue plus direct et une exaltation lyrique du sentir profond dont la mémoire empêchée est la forme éclatée. En effet, le lecteur peut être déstabilisé par le passage d'une écriture plus ou moins réaliste, analytique, narrative, à une écriture métaphorique parsemée d'associations, de vision, de ruptures, d'opposition par le biais d'une syntaxe palpitante dont l'expression liturgique s'exprime dans le Sankofa :

Nous sommes cette région du monde, aussi vaste que méconnue.

Nous sommes l'insu de tous.

Nous sommes la dette envers soi-même, qui ne peut être remise.

Nous sommes l'accablement intime, le tumulte secret...

Nous sommes le cri de San Ko Fa, qui dit que le passé le plus amer ne peut être ignoré (ibid., 123). ${ }^{15}$

18 Ainsi la diatribe des "sans sépulture » conduit à une attitude dialogique et osmotique d'avec les agissements des vivants. La pression morts-vivants comme co-présence des univers narratifs ne fusionne que lorsqu'enfin une cérémonie symbolique est dédiée aux défunts péris dans l'anonymat : la cérémonie de Sankofa, à la fois, symptôme et guérison d'une part, rituel symbolique pour le Souvenir et devoir éthico-politique de la mémoire d'autre part. Pour un socio-diagnostic aux fins d'une catharsis collective et pour soigner ce traumatisme commun, comme Fanon, nous proposons une "socialthérapie " (Fanon, 2002 : 7), [un traitement thérapeutique appliqué aux patients vivant dans un état psychotique de dépersonnalisation absolue], pour célébrer la mémoire retrouvée :

Assise face à eux tous, elle faisait entendre la voix des esprits, des disparus. Elle voyait au-delà du visible, traversait le temps. (...) (Miano, 2009 : 199). Epupa avait 
demandé qu'on l'aide à préparer un bain. Les personnes présentes devraient se laver (...) elle avait expliqué que c'étaient les premières étapes d'un rituel de purification. (...) (ibid. : 219-220). Ayané participa, pour la première fois, à un rituel associant tous les membres de la communauté. (...) Le rituel ne modifiait pas son histoire, n'altérait pas sa personnalité. Pourtant, il donnait du sens à tout cela. Elle se sentait apaisée, rassemblée. Les épaisseurs de son identité formaient une cohérence (ibid. : 244-245-249). souffrance qui dé-/peint le tragique des peuples subsahariens en déphasage avec ses propres incohérences. C'est pourquoi les personnages identifiés comme fous demeurent dans l'obscurité jusqu'à ce qu'ils tissent le savoir inconscient qui les fait agir. En revanche, la société s'écroule de façon pathétique et carnavalesque sous le poids de normes (aliénantes) qui empêchent de forme pernicieuse l'exploration lucide des abîmes d'une histoire ignorée.

21 En effet, aussi bien la société que les «fous déclarés » et les « déclarés fous » de par leurs démarches subversives comme Epupa, Ayané, Musango, Aïda, etc., ils expriment le même malaise face à leur "non-histoire " à des degrés différents. Les différentes formes de délires sont probablement contrapuntiques et dialogiques. Nous avons vu que le délire de théâtralisation met en scène une figure principale tandis que le délire de représentation se trouve dissimulé et «infesté » dans divers dispositifs persuasifs aiguillonnés par une idéologie dominante bien qu'erronée. Cependant toutes deux sont une forme paroxystique d'un délire collectif brillamment mis en contexte pour prendre la mesure d'une amnésie féconde dont l'écho trouve sa répercussion dans le collimateur-catalyseur d'une mémoire retrouvée. À ce titre, la "défocalisation » du discours idéologique en vigueur ainsi que la décongestion du dispositif narratif -rendues possibles grâce à la mise en évidence du "syndrome Sankofa » et ses subséquentes anachronies - suggèrent que seul un délire verbal peut offrir divers axes de lecture afin de conduire le lecteur dans l'œil du cyclone. mémoire, une démarche émancipatrice salutaire devrait viser à décoloniser les imaginaires postcoloniaux non seulement en Occident mais aussi en Afrique subsaharienne. Si la fiction est d'une part, une utopie prise dans le sens de «la plus belle contestation de ce qui est » et d'autre part, une capacité d'affabulation pour proposer autre chose au-delà de l'existant, c'est-à-dire l'expérimentation des possibles, les romans de Miano viennent infléchir l'inertie collective pour s'inscrire dans une dynamique subversive de réinterprétation des idéotypes à l'œuvre dans les mécanismes des représentations mémorielles en région subsaharienne, notamment autour de l'esclavage et de la Traite Négrière. Une démarche somme toute émancipatrice car l'on s'affranchit des didascalies et divers schémas actanciels où la coresponsabilité de la Traite Négrière à partir de l'Afrique par/pour les Africains n'est envisagée que comme une aberration nauséabonde ou l'anti-face d'un négationnisme pernicieux. C'est dans ce contexte que l'historien et universitaire sénégalais Ibrahima Thioub expliquait le contexte d'élaboration des divers récits nationaux dans un article récent intitulé « Esclavage : Les responsabilités africaines dans la traite atlantique des Noirs ». 
ub expose que « les universitaires africains qui participent à l'élaboration d'un savoir académique sur le continent le font surtout au lendemain de la Seconde Guerre mondiale. Cette époque est celle de l'expansion et de l'apogée d'un mouvement anticolonial et nationaliste qui avait besoin d'unifier l'ensemble des Africains contre le pouvoir colonial. Il n'était donc pas question de mettre le doigt sur les éléments qui divisent ou de concevoir les sociétés africaines comme des sociétés hiérarchisées qui ont leurs propres systèmes de domination interne ». Cependant, en occultant ces systèmes de domination internes, on ne comprend hélas qu'à demi l'impact négatif de la Traite sur les sociétés africaines et ses conséquences désastreuses sur le travail de mémoire. Car, en s'interdisant d'interroger leur histoire pour en comprendre les mécanismes de fonctionnement socioculturel et politique, ces chercheurs se terrent dans des binarités manichéennes quelque peu épuisées "Blancs-Noirs ", "Europe-Afrique», "victimesbourreaux». L'on sait que «l'histoire savante, notamment l'écriture de l'histoire nationale, n'est pas une démarche vierge de toute fonction sociale ou identitaire. Elle charrie elle aussi de l'idéologie» (Henry Rousso, 1998: 24). C'est pourquoi, la folie symbolique d'Epupa, une fois décortiquée, expose en réalité les conséquences néfastes de l'exclusion des "sans sépulture " dans les représentations mémorielles à l'œuvre en Afrique subsaharienne. Or, on a pu constater que le trinôme « Epupa, Sankofa et le délire de théâtralisation » ne font qu'un et exhument rétrospectivement l'amnésie collective ayant pris d'assaut les récits nationaux sur le débat autour de l'esclavage et ses impacts. Une alternative à cette obstruction serait le recours à des contre-narrations (même périphériques) visant à enrayer l'ordre narratif agissant autour d'un seul récit. Peut-être à cet instant, en procédant par disruption, on en aura fini avec la tradition du silence qui se chevauche avec argutie avec la mémoire empêchée.

\section{BIBLIOGRAPHIE}

CHAMOISEAU, Patrick (1997). Ecrire en pays dominé. Paris : Gallimard.

ETOKE, Nathalie (2010). Melancholia Africana. L'Indispensable Dépassement de la condition noire. Paris : Editions du cygne.

FANON, Frantz (1961, 1968/2002). Les damnés de la terre. Paris : La Découverte/Poche.

GENETTE, Gérard (1972). Figures III. Paris : Seuil.

GLISSANT, Edouard (2002). Le Discours antillais. Paris : Gallimard.

GLISSANT, Edouard (1975/1997). Malemort. Paris : Gallimard.

GLISSANT, Edouard (1990). Poétique de la Relation. Poétique III. Paris : Gallimard

KRISTEVA, Julia (1988/2007). Étrangers à nous-mêmes. Paris : Gallimard.

MBEMBE, Achille (mars 2000). “À propos des écritures africaines de soi”. Politique africaine, $\mathrm{n}^{\circ} 77$.

MEMMI, Alfred (1985). Portrait du colonisé (précédé de Portrait du colonisateur et d'une préface de Jean-Paul Sartre, Agence de coopération culturelle et technique, Francopoche. Paris : Gallimard. 
MIANO, Léonora (2005). L'Intérieur de la nuit. Paris : Plon.

MIANO, Léonora (2006). Contours du jour qui vient. Paris : Plon.

MIANO, Léonora (2009). Les Aubes écarlates « Sankofa Cry». Paris : Plon.

MIANO, Léonora (2013). La Saison de l'ombre. Paris : Grasset.

REY, Jean-Michel (2010). L'Oubli dans les temps troublés. Paris : Les Éditions de l'Olivier.

RICOEUR, Paul (2000). La Mémoire, l'histoire, l'oubli. Paris : Seuil.

RoBIN, Régine (2003). La Mémoire saturée. Paris : Stock.

Rousso, Henry (1998). La Hantise du passé. Paris : Textuel.

SALMON, Christian (2007). Storytelling la machine à fabriquer des histoires et à formater les esprits.

Paris : La Découverte.

SARTRE, Jean-Paul (1943/2011). L'être et le néant. Essai d'ontologie phénoménologique. Paris :

Gallimard.

STORA, Benjamin (2001). La Guerre invisible: Algérie, années 90. Paris : Presse de Science Po.

THIOUB, Ibrahima (novembre 2016). « Esclavage : Les responsabilités africaines dans la traite

atlantique des noirs »<URL : http://www.gauchemip.org/spip.php?article6754 > (consulté le 21

novembre 2016).

TODOROV, Tzvetan (2008). Les Abus de la mémoire. Paris : Arléa.

\section{NOTES}

1. Rancière : « le concept d'anachronisme et la vérité de l'historien », L'Inactuel, numéro 6, 1996, p. 67-68, cité par http://www.fabula.org/atelier.php?Anachronisme_et_anachronie. Voir aussi <URL : http://www.cnrtl.fr/definition/anachronie (toutes les pages consultées le 07/12/2016)>

2. Les notions de rétrospective (analepse) et anticipation (prolepse) sont à analyser comme souscatégories des anachronies.

3. Nous analyserons les catégories du temps où les infidélités chronologiques s'inscrivent dans une dynamique dialogique entre les référents historiques réels rapportés dans le texte et leur signifié romanesque.

4. Glissant énumère quatre formes essentielles de délire verbal coutumier: délire de communication, de théâtralisation, de représentation et de persuasion. Notre approche sera principalement axée sur le délire de théâtralisation bien que nous pourrons mentionner les autres formes de délire de façon sporadique. Glissant explique que le délire verbal « coutumier » s'illustre par « toute manifestation subversive, insubordonnée ou déviante d'un langage (écrit ou parlé) en référence à une norme standardisée, idéale ou réelle, de plus grande usance au sein d'une société ». Lire aussi à ce propos, Patrick Chamoiseau qui analyse la conséquence des rapports de domination mémorielle dans Ecrire en pays dominé, 1997).

5. Le délire de théâtralisation est une forme de représentation scénique de la praxis verbale représentée par un individu comme pulsion dramatique et commune d'un groupe relativement homogène. Cet individu est donc un acteur en scène, pour toute la communauté qui se trouve en même temps comme spectateur et acteur autoréflexif. Ici, l'évidence est secrète au début. Les processus se trouvent par accumulation, consécution et formule. (Glissant : 2002, 646-651).

6. Dans La mémoire, l'histoire, l'oubli (2000), Paul Ricœur observe trois types de pathologies de la mémoire: la mémoire empêchée -(objet de notre étude)-, inspirée directement de la 
psychanalyse ; la mémoire manipulée, instrument et bastion des récupérations idéologiques ; la mémoire abusivement commandée ou obligée comme lieu de référence éthico-politique du devoir de mémoire (689). On pourrait aussi lire le classique Portrait du colonisé... d'Alfred Memmi, un écho des travaux de Frantz Fanon sur les disfonctionnements de la mémoire chez le colonisé.

7. En partant de Das Unheimliche (1919) de Freud, Julia Kristeva (1988/2007 : 269-283) analyse l'ambivalence de l'inquiétante étrangeté à travers les termes allemands : « Heimlich/unheimlich ». Il semble que l'adjectif « heimlich » renferme un sens proche de son antonyme « unheimlich » : «le familier », «l'intime ». "Le familier » signifierait dans son acception élargie "le secret», «l'occulte », « le ténébreux » ou « le dissimulé ».

8. Ce fragment inspiré d'un poème de Birago Diop intitulé «Souffles». Poème dans lequel le poète présente une cosmovision subsaharienne portée sur l'interconnexion des morts et des vivants.

9. Passage du milieu (ou Middle Passage) est un film martiniquais (de Guy Deslauriers, 2001) qui illustre ces traversées transatlantiques durant lesquelles des esclaves subsahariens périrent dans l'anonymat.

10. C'est nous qui soulignons.

11. Le débat sur la co-responsabilité africaine de la Traite Négrière divise les subsahariens dont la plupart renvoie le débat à un dol ne pouvant être apprécié ni tranché hors contexte : <URL : http://www.slateafrique.com/1933/intellectuels-africains-traite-negriere-responsabilite (consulté le 15/11/2011) et Achille Mbembé dans "À propos des écritures africaines de soi" (Politique africaine, $\mathrm{n}^{\circ} 77$ - mars 2000).

12. C'est nous qui soulignons.

13. C'est nous qui soulignons.

14. C'est nous qui soulignons.

15. C'est nous qui soulignons.

\section{RÉSUMÉS}

L'article vise à analyser le concept du «Sankofa » illustré par Epupa, le personnage identifié comme fou dans les premiers romans de Léonora Miano. Pour ce faire, nous nous inspirons de la théorie du « délire de théâtralisation » de Glissant. Ce délire serait l'émanation symptomatique d'une amnésie collective à l'œuvre dans des sociétés anciennement colonisées. Pour comprendre les enjeux sociopolitiques de l'oubli en Afrique subsaharienne, entre devoir de mémoire, travail de mémoire, carence de mémoire, abus de mémoire et déficit critique, une démarche émancipatrice qui passe par la réélaboration de canons mémoriels inclusifs sera suggérée en guise de conclusion pour décoloniser/décloisonner les imaginaires transcontinentaux aussi bien en Afrique subsaharienne qu'en Occident.

The purpose of our work is to analyze the concept of « Sankofa " illustrated by Epupa, the character identified as mad in Léonora Miano's first novels. In this respect, our analysis will stem from Glissant's theory of «theatrical delusion ». This delirium would be the symptomatic emanation of a collective amnesia at work in formerly colonized societies. In order to understand the socio-political stakes of oblivion in sub-Saharan Africa, between the duty of memory, memory work, lack of memory, abuse of memory and critical deficit. An emancipatory approach that involves the re-elaboration of inclusive memory models will be suggested in our conclusion 
to decolonize / decompartmentalize the transcontinental imaginary as much in sub-Saharan Africa as in the West.

INDEX

Mots-clés : littérature francophone, Sankofa, anachronie, histoire, oubli

Keywords : francophone literature, Sankofa, anachrony, history, forgetfulness

\section{AUTEUR}

FERDULIS ZITA ODOME ANGONE

Université Cheikh Anta Diop de Dakar

odomezita[at] yahoo.fr 